\title{
Ventajas y limitaciones de la citogenética en la medicina actual
}

\author{
*Elodia Torres \\ * Laboratorio de Citogenética. Departamento de Genética del Instituto de Investigaciones en Ciencias de la Salud. \\ Universidad Nacional de Asunción. San Lorenzo, Paraguay
}

\author{
Cómo referenciar este artículo/ \\ How to reference this article:
}

Torres E. Ventajas y limitaciones de la citogenética en la medicina actual. Mem. Inst. Investig. Cienc. Salud. 2018; 16(2): 107-112

\section{R E S U M E N}

La citogenética es el estudio de los cromosomas tanto en número como en estructura. En 1882 Flemming publica las primeras primeras ilustraciones de los cromosomas humanos a partir de observaciones al microscopio y recién en el año 1953, Tjio y Levan determinaron el número real de cromosomas humanos por célula diploide $(2 n=46)$. El propósito de este trabajo es presentar el valor, uso actual e importancia de los estudios citogenéticos en aquellos casos en que el profesional de salud se enfrente a un paciente con una probable enfermedad de causa genética o síndrome dismórfico, además de exponer algunas experiencias de un laboratorio de Citogenética en el Paraguay, donde se realiza el estudio cromosómico. Aún con el advenimiento de la Biología Molecular y de la Citogenética Molecular, la citogenética convencional sigue siendo una herramienta de gran importancia, ya que permite realizar el diagnóstico de una enfermedad genética en pacientes con sospecha clínica de ser portadores de anomalías cromosómicas, y por tanto asesorar a las familias respecto de dicha enfermedad, proveer un pronóstico, riesgo de recurrencia y en casos que se requiera, un tratamiento.

Palabras clave: cromosomas humanos, citogenética, estudio genético, diagnóstico.

\section{Advantages and limitations of cytogenetics in current medicine}

\section{A B S T R A C T}

Cytogenetics is the study of chromosomes both in number and structure. The first publications about human cytogenetics were provided towards the end of the 19th century with the publication of Flemming in 1882 of the first figures of human chromosomes from observations under the microscope and only in 1953, Tjio and Levan determined the actual number of human chromosomes per diploid cell $(2 n=46)$. The purpose of this paper is to present the value, current use and importance of cytogenetic studies in those cases in which the health professional faces a patient with a probable disease of genetic causes or dysmorphic syndrome, in addition to exposing some experiences from a Cytogenetics laboratory in Paraguay, where chromosomal study is carried out. Even with the arrival of Molecular Biology and Molecular Cytogenetics, conventional cytogenetics is a tool with a great importance, which allows the genetic disease diagnosis in patients with clinical suspicion of being carriers of chromosomal abnormalities, allowing to advice families about the disease, as well as to provide a prognosis, risk of recurrence and, in cases that requires it, a treatment.

Keywords: human chromosomes, cytogenetics, genetic study, diagnosis.

\section{DESARROLLO}

La citogenética clásica o convencional constituye una herramienta insustituible tanto en los estudios prenatales, postnatales como en investigaciones relacionadas al cáncer ${ }^{(1)}$. Se utiliza para el diagnóstico de enfermedades genéticas de origen cromosómico, permite la Fecha de recepción: febrero 2018. Fecha de aceptación: marzo 2018

*Autor correspondiente: Elodia Torres. Universidad Nacional de Asunción, Instituto de Investigaciones en Ciencias de la Salud, Laboratorio de Citogenética. Departamento de Genética. San Lorenzo, Paraguay 
detección de patologías cromosómicas asociadas a retardo mental, malformaciones congénitas múltiples y enfermedades de origen hemato-oncológicos (2). A finales del siglo XIX Flemming publica las primeras ilustraciones del cromosoma humano a partir de observaciones en el microscopio. En 1921 Painter demostró la presencia del cromosoma Y en preparaciones obtenidas a partir de testículo y reveló que el número total de cromosomas era de 48 (Fig. $1^{\text {)(3) }}$. A raíz de estos descubrimientos, habría surgido el interrogante si el sexo en los humanos estaba determinado mediante $\mathrm{XO}$ o XY.

Sin embargo, el gran desarrollo de la citogenética se inició con la determinación del número de cromosomas en el cariotipo humano, que era 46 , y de que los cromosomas que estaban implicados en la determinación del sexo eran iguales en la mujer $(X X)$ y diferentes en el varón (XY), a éstos se les llamó cromosomas sexuales o gonosomas, mientras que a los 22 pares restantes se les denominó autosomas ${ }^{(4-5)}$. En el congreso de citogenética en Denver en 1960, se propuso ordenar a los 46 cromosomas de acuerdo a su longitud numerando los pares del 1 al 23. Ese mismo año, Patau se opuso a esta simple clasificación demostrando que algunos cromosomas no se podían clasificar inequívocamente sólo por su longitud y posición del centrómero, posteriormente se aprobó subdividir los pares de cromosomas en grupos, incluyendo en cada uno aquellos pares similares en su morfología(6).

Precisamente, el cariotipo es la agrupación y organización de los cromosomas de acuerdo a su tamaño y posición del centrómero. El número asignado a cada cromosoma está basado en el patrón de Bandas Q como fuera propuesto por Casspersson y colaboradores en $1971^{(7)}$. Este patrón se obtiene a partir de células en división. Generalmente se utiliza sangre periférica para la obtención de las preparaciones cromosómicas, aunque también puede realizarse a partir de células fetales de líquido amniótico, vellosidades coriales y médula ósea, entre otros. Igualmente según la posición del centrómero un cromosoma presenta un brazo corto (p) y un brazo largo (q) y se clasifican en metacéntricos, cuando los brazos son de igual tamaño, submetacéntricos, cuando los brazos son desiguales y acrocéntricos cuando el brazo corto está modificado en un satélite ${ }^{(8)}$.

Las patologías cromosómicas, se deben principalmente a las alteraciones en el cariotipo, estas pueden deberse a una variación en el número normal de cromosomas o a cambios en su estructura. Si la alteración implica un exceso o un defecto de material genético se denomina desequilibrada y produce mstalformaciones congénitas, con frecuencia acompañadas de retraso mental, sin embargo, una alteración equilibrada no produce, en principio, enfermedad en el individuo portador, aunque sí hay riesgo en su descendencia ${ }^{(3-}$ 5).

El propósito de este trabajo es presentar una breve reseña de lo que significa la citogenética desde sus comienzos hasta nuestros días, describir las principales técnicas citogenéticas que se realizan en el laboratorio de Citogenética del IICS y a nivel global, analizando sus implicaciones en la práctica clínica.

\section{Técnica de Citogenética Clásica o Convencional}

Para el estudio citogenético, se efectúa un procedimiento estándar, desde la extracción de sangre periférica del paciente seguido de un cultivo celular de linfocitos en medios especiales con un mitógeno y un compuesto químico que detiene la división celular en metafase. Posteriormente se realiza una "cosecha" que consiste en el procesamiento de la muestra con una solución hipotónica, sucesivos lavados con fijadores, hasta la obtención de un extendido, el cual es sometido a distintas técnicas de tinción. En primer lugar, directamente con el colorante de Giemsa para su tinción convencional, con esta técnica se realiza un recuento para determinar el número de cromosomas del paciente, normalmente se observan 20 a 30 metafases, pero en casos especiales, como mosaicismos o búsqueda del cromosoma $X$ frágil, se registran de 50 a 100 metafases, con esta tinción no es posible la identificación individual de cada cromosoma, se puede llegar hasta la determinación del sexo. En segundo lugar, se tratan los preparados con técnicas de Bandeo $\mathrm{G}^{(7)}$, con esta técnica se logran visualizar anomalías visibles al microscopio óptico. La otra técnica que se realiza en el laboratorio son las bandas $\mathrm{C}$, consiste en poner de manifiesto la heterocromatina constitutiva tanto centromérica como pericentromérica y gran parte del cromosoma $Y$ a un nivel telomérico ${ }^{(1)}$. Con esta técnica se identifican a los pares cromosómicos 1, 9, 16 e Y. Finalmente para la realización del resultado del paciente, se obtiene el cariotipo, el cual consiste en agrupar a los cromosomas de a pares, a través del 
software "Videotest-Karyo 3.0", que constituye un soporte informático adecuado ${ }^{(8)}$. Además de los linfocitos de sangre periférica, la otra muestra que se cultiva en nuestro laboratorio es el de médula ósea para el diagnóstico de enfermedades hemato-oncológicas, el procesamiento de la misma es similar al de linfocitos, con algunos cambios principalmente en el tiempo de cultivo.

\section{El Cariotipo: "un mundo fascinante de cromosomas"}

El cariotipo permite analizar los cromosomas de una célula en metafase, agruparlos por pares idénticos y clasificarlos según su morfología y tamaño. En la confección del cariotipo se agrupan por tamaño y posición del centrómero, desde la letra A hasta la G. En el grupo A se encuentran los pares cromosómicos de mayor tamaño, el 1,2 y 3 , los pares 1 y 3 son metacéntricos y el par 2 submetacéntrico. En el grupo B se ubican a los pares 4 y 5 que son submetacéntricos grandes, mientras que en el grupo $C$ se ubican desde el par 6 hasta el 12 incluido el cromosoma sexual " $X$ ", todos submetacéntricos de tamaño mediano. En el grupo D se localizan los pares 13,14 y 15, que son los acrocéntricos grandes con satélite; en el grupo E se ubican los pares 16,17 y 18 , el par 16 es metacéntrico mediano y los pares 17 y 18 son submetacéntricos pequeños. En el grupo $F$ se localizan los pares 19 y 20, metacéntricos pequeños y en el grupo $G$ están localizados los pares 21,22 y el cromosoma "Y", todos acrocéntricos pequeños con satélite, el" $Y$ " carece de satélite ${ }^{(8)}$

En la figura 1 se observa la metafase publicada por Painter en 1921, donde se señala el cromosoma " $Y$ ", por entonces se consideraba que el número cromosómico era de 48 (Figura 1). En la figura 2 se observa la metafase presentada por primera vez por Tjio y Levan en 1956, (Figura 2), fue a partir de entonces que se describió el número normal de cromosomas humanos, el cual se confirmó era de $46^{(2,3)}$. En las Figuras 3,4 y 5 , se observan metafases con las diferentes técnicas que se utilizan en nuestro laboratorio, con tinción convencional (Figura 3), metafase con bandas G (Figura 4), y con bandas C (Figura 5). Las Figuras 6 y 7 denotan "los cariotipos" que se obtienen a partir del software Videtotest-karyo 3.0, con los que se elabora el resultado final del paciente.

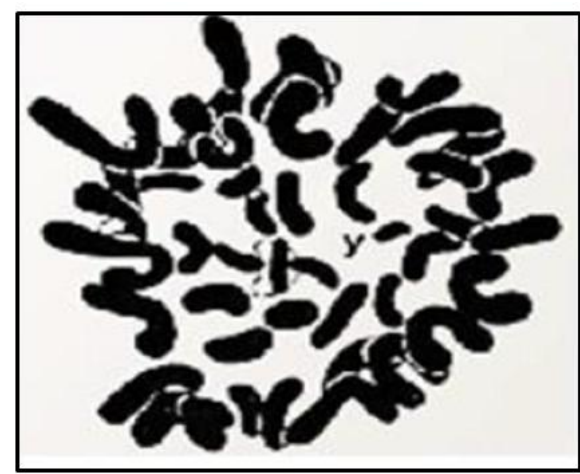

Figura 1: Metafase obtenida por Painter en 1921 donde se observa el cromosoma "Y".

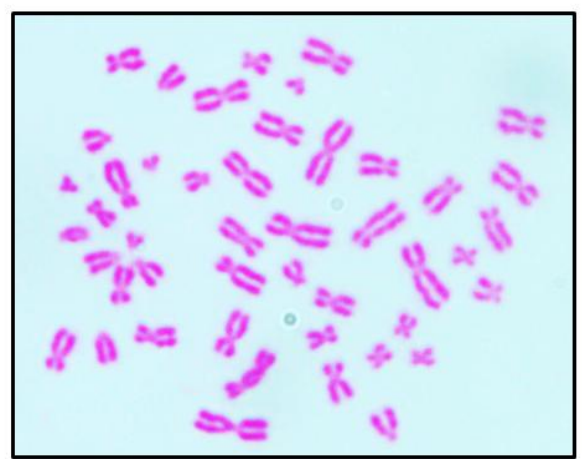

Figura 3: Metafase con técnica de tinción convencional obtenida actualmente.

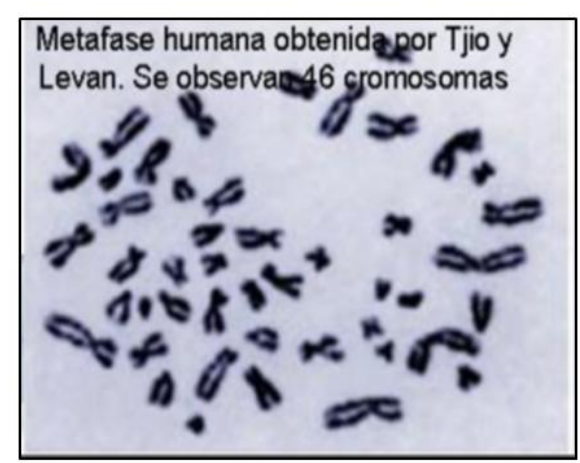

Figura 2: Metafase observada por Tjio y Levan en 1956.

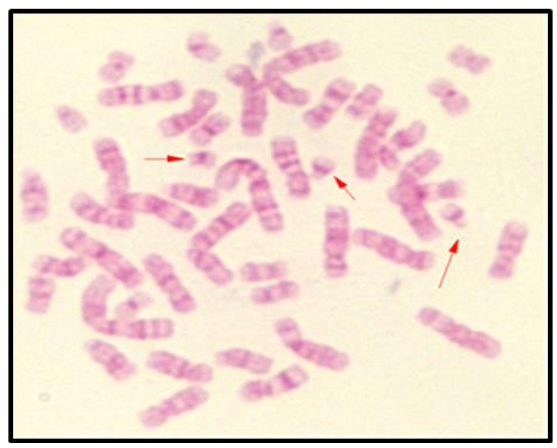

Figura 4: Metafase con técnica de Bandas $\mathrm{G}$ con 550 bandas 


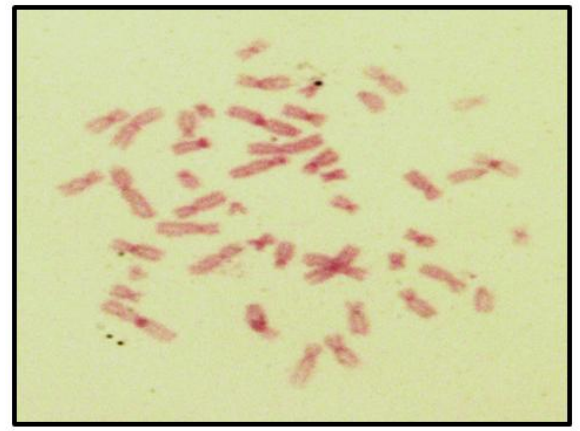

Figura 5: Metafase con Bandas $C$, técnica que tiñe la heterocromatina constitutiva.

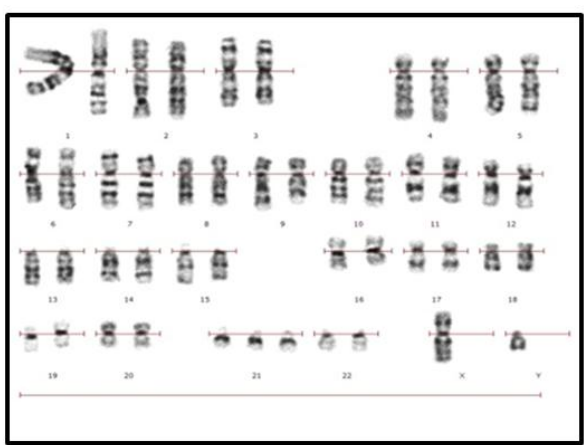

Figura 7: Cariotipo obtenido con el software, para el resultado final del paciente, en esta imagen se observa una trisomía del cromosoma 21.

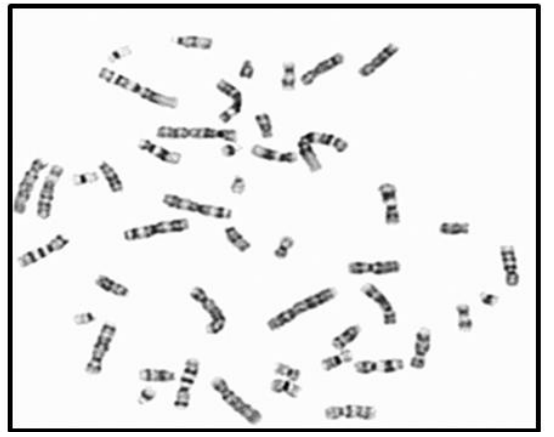

Figura 6: Metafase obtenida con el software Videotest-karyo 3.0

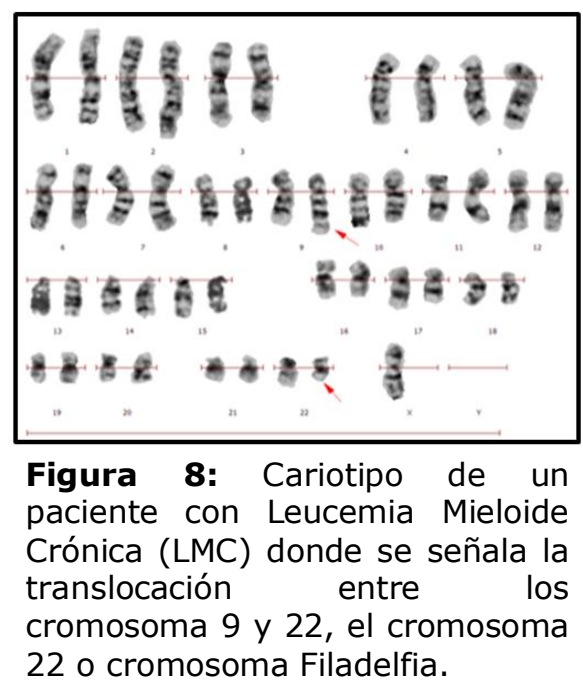

\section{Técnicas de Citogenética Molecular}

La hibridación in situ fluorescente (FISH), es una técnica citogenética molecular que se utilizó por primera vez para determinar la localización cromosómica de secuencias particulares de ADN, y es una técnica que se aplica a células no cultivadas en interfase para la detección rápida de aneuploidías, en células en metafase para la detección de síndromes de microdeleción, caracterización de reordenamientos cromosómicos poco marcados e identificación de marcadores cromosómicos que no pueden identificarse mediante las técnicas citogenéticas clásicas ${ }^{(1)}$. Esta técnica se utiliza para determinar e identificar rearreglos cromosómicos crípticos, microdeleciones, microduplicaciones y para identificar un material adicional de origen desconocido, que con las técnicas actuales que se tienen en el laboratorio no es posible observar. La gran ventaja de esta técnica es que la secuencia que se investiga puede observarse tanto en metafase como en interfase, el resultado del paciente se obtiene al cabo de 24 a 48 horas. La gran desventaja es que solo se identifica lo que se busca sin que proporcione información sobre el resto del genoma, además de los costos elevados $^{(1,11,12)}$. La metodología FISH se basa en la complementariedad de las dos cadenas de ADN de doble hélice; en primer lugar, la muestra de ADN, ya sean cromosomas en metafase o núcleos en interfase, se desnaturalizan, posteriormente se le añade la sonda de interés marcada con un fluoróforo que se asocia al ADN de la muestra en el sitio diana, en un proceso denominado hibridación, se vuelve a formar la doble hélice, la señal emitida por la sonda se observa en un microscopio de fluorescencia y así la muestra se clasifica según la presencia o ausencia de la señal. La incorporación de esta técnica a nivel país se realizará en futuro próximo a corto plazo, precisamente en este momento, esta técnica está siendo ejecutada a través de un proyecto de investigación financiado por el Consejo Nacional de Ciencia y Tecnología. 


\section{"Los reportes de Casos Clínicos"}

A nivel global, con el bandeo $\mathrm{G}$ se ha establecido que, 1 de cada 150 recién nacidos vivos, $50 \%$ de los abortos del primer trimestre y $20 \%$ de los de segundo, $20 \%$ de los ovocitos, $4 \%$ de los espermatozoides, $10 \%$ de las concepciones, $8 \%$ de los mortinatos y $4 \%$ de las parejas infértiles tienen una alteración cromosómica ${ }^{(11,12)}$. En Citogenética ocurre una particularidad muy interesante en el mundo de las ciencias de la salud. Como ya se ha mencionado más arriba, los pacientes que se realizan el estudio citogenético son personas con sospecha clínica de ser portadores de una enfermedad genética de causa cromosómica, para lograr un diagnóstico de esta patología se requiere de una persona altamente capacitada y especializada, lo cual conlleva años de entrenamiento, pues el análisis cromosómico se realiza a través de un microscopio óptico, tanto para la técnica convencional como las de Bandeo cromosómico. En primer lugar se realiza el recuento de cromosomas a fin de descartar una anomalía cromosómica numérica, en segundo lugar, con las técnicas de bandeo, se analizan cada uno de los 23 pares de cromosomas en forma individual. De esta manera, un paciente puede ser considerado un "un caso clínico de investigación", pues depende del ojo del observador la detección de la anomalía cromosómica para el logro del resultado de certeza, y por tanto del diagnóstico, incluso en muchas ocasiones se recurre a la literatura para determinar el tipo de anomalía cromosómica, en pacientes portadores de retardo mental y dismorfias, para lo cual se recurre al "OMIM - Online Mendelian inheritance in Man, An Online Catalog of Human Genes and Genetic Disorders" y para las enfermedades hemato-oncológicas al "Atlas of Genetics and Cytogenetics in Oncology and Haematology"(13,14).

El valor de los casos clínicos resueltos por citogenética se centran en primer lugar la baja frecuencia de este tipo de enfermedad y la tasa de mortalidad en pacientes portadores de una anomalía cromosómica en muy elevada. En segundo lugar, en el tiempo que requiere la finalización del resultado a través del análisis minucioso de cada uno de los 23 pares de cromosomas. A esto se suma la expresión fenotípica muy variada en estos pacientes, lo que dificulta en muchas ocasiones llegar a un diagnóstico de certeza.

\section{CONSIDERACIONES FINALES}

De esta manera, entre las enfermedades genéticas de origen cromosómico, ocupan el primer lugar las anormalidades numéricas, en las que están afectadas la cantidad de cromosomas del individuo, y entre éstas, las aneuploidías autosómicas son las más frecuentes en los nacidos vivos, como la Trisomía 21 o Síndrome de Down, la trisomía 18 o Síndrome de Edwards, la trisomía 13 o Síndrome de Patau, y las aneuploidías que involucran a los cromosomas sexuales, como el Síndrome de Klinefelter, que son varones con un cromosoma $X$ demás y la monosomía del cromosoma $X$, la única viable en niñas con Síndrome de Turner. En segundo lugar, se tienen a las enfermedades cromosómicas estructurales que se clasifican en desequilibradas y equilibradas. Entre las primeras se tienen las deleciones con o sin formación de anillo, inserciones, duplicaciones e isocromosomas, y entre las equilibradas, se tienen las inversiones y las translocaciones. En este grupo de anomalías estructurales se tiene una amplia gama de posibilidades que incluyen pequeñas deleciones denominadas microdeleciones como en el Síndrome de WolfHirschorn, en la que está presente una deleción de una porción del brazo corto del cromosoma $4(4 \mathrm{p}-)$, el Síndrome de DiGeorge que es deleción del brazo largo del cromosoma 22 (del22q11.2) y otras con pérdida de mayor cantidad de genes involucrados, como el Sindrome de Cri-du-chat, en la que está delecionado el brazo corto del cromosoma $5\left(5 \mathrm{p}^{-)(2,8,11,12)}\right.$. En otro grupo importante, en donde la citogenética es una muy útil como herramienta diagnóstica, se encuentran las enfermedades hemato-oncológicas que presentan en su mayoría anomalías cromosómicas clonales. Los registros de citogenética de los diferentes tipos son regularmente publicados en el Catalog of Chromosomes Aberrations in Cancer, que en su quinta edición ya tiene descritas anomalías cromosómicas de más de 22.000 neoplasias $^{(14)}$.

Las indicaciones para la realización del cariotipo que se utilizan son principalmente, las malformaciones congénitas, retardo del desarrollo sicomotor, retardo mental de origen desconocido, talla baja en estudio, ambigüedad genital, padres de niños con alteraciones cromosómicas estructurales y enfermedades hemato-oncológicas, imprescindibles en médula ósea de síndromes mielodisplásicos ${ }^{(6,14)}$. 
A pesar del avance de la biología molecular y de la citogenética molecular, la citogenética clásica o convencional sigue siendo una valiosa herramienta diagnóstica para una gran cantidad de pacientes con múltiples dismorfias y malformaciones, con problemas de esterilidad e infertilidad, los que en algunos casos sugieren un síndrome conocido y en otros, debido a la gran variabilidad en el fenotipo y la severidad de las malformaciones, pueden corresponder a alteraciones tan diversas como las posibilidades de reordenamientos cromosómicos, lo que hace que el estudio cromosómico en estas patologías sean un desafío para el médico especialista. Los estudios cromosómicos son necesarios para establecer el diagnóstico y pronóstico no sólo de los síndromes dismórficos, sino también en las enfermedades hemato-oncológicas malignas, para el seguimiento del paciente y valorar la eficacia de un tratamiento.

Por lo tanto, el estudio cromosómico es una herramienta que permite la confirmación del diagnóstico para dichas enfermedades y de acuerdo al resultado obtenido, se efectúa un adecuado asesoramiento genético del paciente y sus familias.

\section{REFERENCIAS BIBLIOGRAFICAS}

1. Méndez Rosado LA, Nodarse Rodríguez A, Morales Rodríguez $\mathrm{E}$, et al. Diagnóstico prenatal citogenético mediante la hibridación in situ con fluorescencia. Revista Cubana de Ginecología y Obstetricia 2012;38(1):1-10.

2. Gallego MS. Rol de la citogenética en pediatría. Arch Argent Pediatr 2011; 109(4):339-46.

3. Painter TS. The $Y$ chromosome of man. Science. $1921 ; 53: 503-4$.

4. Tjio JH, Levan A. The chromosome number in man. Heredity. 1956; 42:1-6.

5. Ford CE, Hamerton JL. The chromosome of man. Nature 1956; 168:1020-3.

6. Silva C, Contreras N, Fonseca D. Utilidad de la citogenética en la medicina actual. Acta Médica Colombiana 2008; 33(4):309-16.

7. Casspersson $\mathrm{T}$, Feber $\mathrm{S}$, Foley GE, Kudynowsju J. Modest EJ, Simonsson E, et al. Chemical differentiation along metaphase chromosomes. Exp Cell Res 1968: 49:219-22.

8. Shaffer LG, McGowan-Jordan J, Schmid M. ISCN An International for Human Cytogenetic Nomenclature. Published in collaboration with Cytogenetic and Genome Research. Switzerland. 2013.

9. Estay FA, Parra LR, Benítez $\mathrm{CH}$. Alteraciones cromosómicas en linfocitos de sangre periférica. Rev Chil Pediatr 2007; 78(4):363-8.

10. Verma RS, Babu A. Human chromosomes (Manual of basic techniques). Pergamon Press USA. 1989.

11. Thompson MW, Mclnnes RR, Willard HF. Citogenética Clínica: Principios generales y anomalías autosómicas. En: Thompson \&
Thompson. Genética en Medicina. 4a. Edición: Barcelona, España. Masson S.A; 2002. p191218.

12. Aiassa D, Gorlal N. Prevalencia de anomalías cromosómicas en pacientes referidos para diagnóstico citogenético en la Ciudad de Rio Cuarto. Experiencia Médica 2010; 28(1):516.

13. OMIN. Online Medelian Inheritance in Man. An Online Catalog of Human Genes and Genetic Disorders. Updated April 26, 2018. https://www.omin.org/

14. Atlas of Genetics and Citogenetics in Oncology and Hematology. https://atlasgeneticsoncology.org/

15. Santana Hernández EE, Tamayo Chang VJ, Pérez Breff G. et al. Manifestaciones fenotípicas de un caso con sospecha clínica de trisomía parcial de cromosoma 9p. Gaceta Médica Espirituana. 2015; 17(1):68-73

16. Torres E, Rodríguez S, Monjagata N. Monosomía del brazo largo del cromosoma 9 en una paciente con sospecha de Síndrome de Turner. Reporte de Caso. Mem Inst Investig Cienc Salud 2014; 12(2) : 72-3.

17. Torres $E$, Rodríguez $S$, Monjagata N, Fernández S, Herreros MB , Eza G. Trisomía parcial $9 p$ derivada de una translocación recíproca 9,15, materna. Reporte de casos. Rev. Fac. Salud. UDES (Bucaramanga). 2015; 2(2)139-43.

18. Ascurra $M$, Rodríguez $S$, Herreros $M B$, Torres E. Cromosoma 20 en anillo en gemelas monocigotas. Pediatr. Asunción, 2008, 35(2): 101-105. 